\title{
First firm spectral classification of an early-B pre-main-sequence star: B275 in M 17^
}

\author{
B. B. Ochsendorf ${ }^{1}$, L. E. Ellerbroek ${ }^{1}$, R. Chini ${ }^{2,3}$, O. E. Hartoog ${ }^{1}$, V. Hoffmeister ${ }^{2}$, L. B. F. M. Waters ${ }^{4,1}$, and L. Kaper ${ }^{1}$ \\ 1 Astronomical Institute Anton Pannekoek, University of Amsterdam, Science Park 904, PO Box 94249, 1090 GE Amsterdam, \\ The Netherlands \\ e-mail: ochsendorf@strw.leidenuniv.nl; L.Kaper@uva.nl \\ 2 Astronomisches Institut, Ruhr-Universität Bochum, Universitätsstrasse 150, 44780 Bochum, Germany \\ 3 Instituto de Astronomía, Universidad Católica del Norte, Antofagasta, Chile \\ ${ }^{4}$ SRON, Sorbonnelaan 2, 3584 CA Utrecht, The Netherlands
}

Received 14 September 2011 / Accepted 25 October 2011

\begin{abstract}
The optical to near-infrared (300-2500 nm) spectrum of the candidate massive young stellar object (YSO) B275, embedded in the star-forming region M 17, has been observed with X-shooter on the ESO Very Large Telescope. The spectrum includes both photospheric absorption lines and emission features ( $\mathrm{H}$ and $\mathrm{Ca}$ II triplet emission lines, 1st and 2nd overtone $\mathrm{CO}$ bandhead emission), as well as an infrared excess indicating the presence of a (flaring) circumstellar disk. The strongest emission lines are double-peaked with a peak separation ranging between 70 and $105 \mathrm{~km} \mathrm{~s}^{-1}$, and they provide information on the physical structure of the disk. The underlying photospheric spectrum is classified as B6-B7, which is significantly cooler than a previous estimate based on modeling of the spectral energy distribution. This discrepancy is solved by allowing for a larger stellar radius (i.e. a bloated star) and thus positioning the star above the main sequence. This constitutes the first firm spectral classification of an early-B pre-main-sequence (PMS) star. We discuss the position of B275 in the Hertzsprung-Russell diagram in terms of PMS evolution. Although the position is consistent with PMS tracks of heavily accreting protostars $\left(\dot{M}_{\text {acc }} \gtrsim 10^{-5} M_{\odot} \mathrm{yr}^{-1}\right)$, the fact that the photosphere of the object is detectable suggests that the current mass-accretion rate is not very high.
\end{abstract}

Key words. stars: formation - stars: pre-main-sequence - stars: massive - stars: variables: T Tauri, Herbig Ae/Be

\section{Introduction}

Observational and theoretical evidence is accumulating that the formation process of massive stars is through disk accretion, similar to low-mass stars. This persists despite the strong radiation pressure and ionizing power produced by the massive young stellar object (YSO) that may reverse the accretion flow and prevent matter from accreting onto the forming star (e.g., Keto et al. 2006; Krumholz et al. 2009). Given the short mainsequence lifetime of massive stars, the mass accretion rate must be high (up to $\sim 10^{-3} M_{\odot} \mathrm{yr}^{-1}$, Hosokawa et al. 2010) to ensure that the star is not leaving the main sequence before the accretion process has finished.

Evidence of accretion must come from the detection of circumstellar disks, and possibly bipolar jets, as observed around forming low-mass stars (e.g., Appenzeller \& Mundt 1989). Disks and outflows around massive YSO candidates are being reported (e.g., Chini et al. 2004; Kraus et al. 2010; Ellerbroek et al. 2011), but the physical properties of the forming massive stars remain uncertain. The mass of the central object has to be estimated from the emerging flux, and the direct detection of the photospheric spectrum turns out to be very difficult at this early stage of evolution (e.g., Testi et al. 2010).

* Based on observations performed with the ESO Very Large Telescope on Cerro Paranal, Chile, as part of the X-shooter Science Verification program 60.A-9402(A).
Infrared surveys have revealed several hundred candidate massive YSOs, based on luminosity arguments (e.g., Urquhart et al. 2011). A ( $K$-band) spectrum has been obtained for only a few of these (Hanson et al. 1997, 2002; Bik et al. 2006), and they show a red continuum, likely due to hot dust, and an emission-line spectrum that includes $\mathrm{Br} \gamma$ and, often, $\mathrm{CO} 2.3 \mu \mathrm{m}$ bandhead emission. The latter emission can be modeled as being produced by a Keplerian rotating disk surrounding the young, potentially massive star (Bik \& Thi 2004; Blum et al. 2004; Wheelwright et al. 2010).

As massive stars show most spectral features in the UV and optical ranges, the study of their photospheric properties would strongly benefit from extending the spectral coverage as far to the blue as possible. Obviously, extinction by the surrounding gas and dust makes this an observational challenge. Only in rare cases have spectra of candidate massive YSOs been obtained at optical wavelengths. Hanson et al. (1997) obtained optical and near-infrared spectra of candidate massive YSOs in M 17, one of the most massive nearby star-forming regions in the Galaxy (Hoffmeister et al. 2008; Broos et al. 2007; Povich et al. 2009). For the "normal" OB stars Hanson et al. (1997) found a good correspondence between the optical and $K$-band spectra, but the massive YSO optical spectra remained inconclusive. For four massive YSO candidates, they registered the optical spectrum from 400 to $480 \mathrm{~nm}$, indicating a high mass and luminosity. The blue spectrum of the strong CO emission source B275 showed 

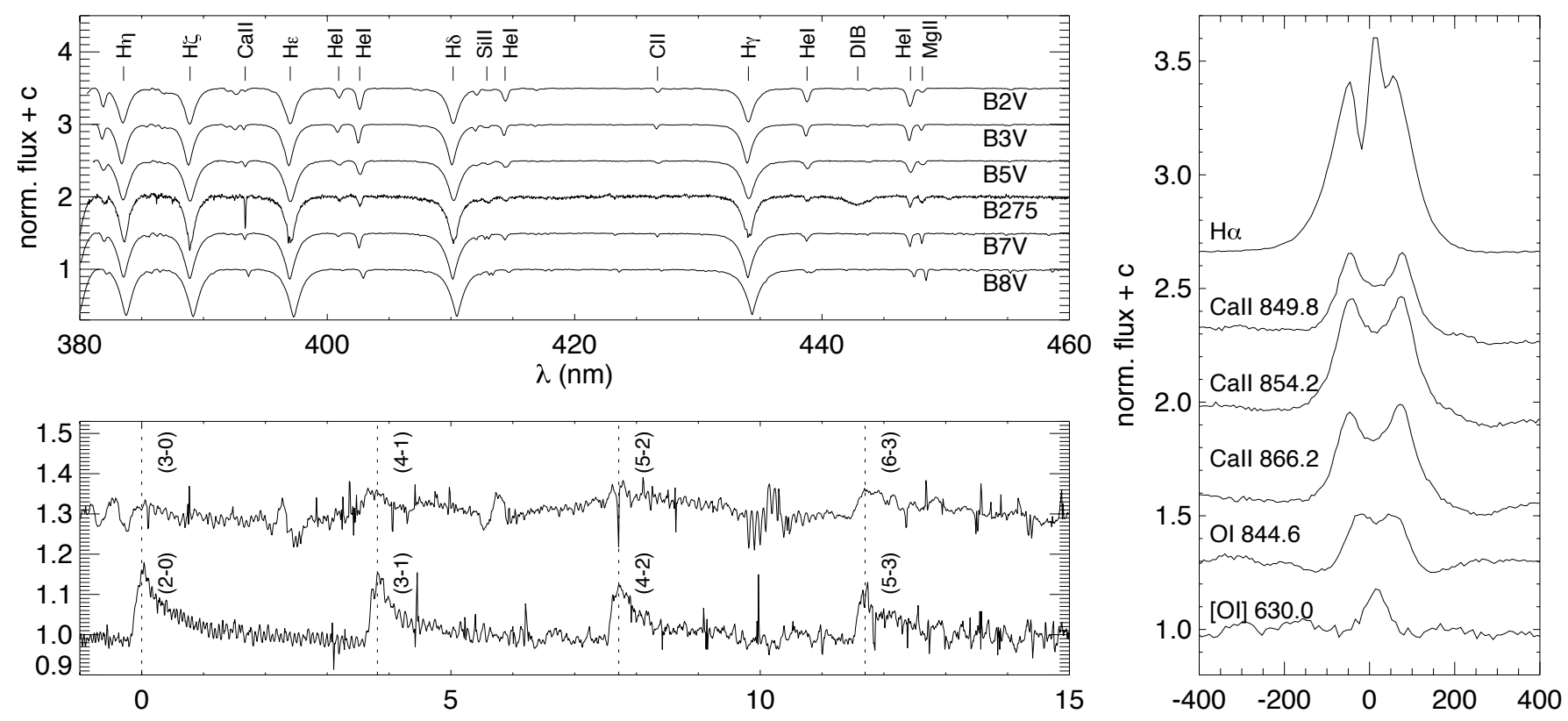

Fig. 1. Top left: the blue spectrum of B275 in M 17 shown next to B main-sequence-star spectra (Gray \& Corbally 2009). Bottom left: the 1 st and 2nd overtone CO emission bands. Zero velocity corresponds to the first component in the series (at 2294 and $1558 \mathrm{~nm}$, respectively). Right: a sample of the emission line profiles in the spectrum of B275. The Ca II triplet lines and O I $845 \mathrm{~nm}$ are superposed on hydrogen Paschen series absorption lines. The flux of the $\mathrm{H} \alpha$ line is scaled down by a factor 5; the structure near the peak is a remnant of the nebular-line subtraction.

no definite photospheric features other than hydrogen, so that the nature of this source remained uncertain. The spectral energy distribution (SED), though, indicated spectral type late-O or early B, at an adopted distance of $1.3 \mathrm{kpc}$. We set out to exploit the high efficiency and broad wavelength coverage of the new medium-resolution spectrograph X-shooter on the ESO Very Large Telescope (VLT) to (i) detect the photospheric spectrum of B275 in M 17; (ii) determine its effective temperature in order to place the candidate massive YSO unambiguously onto recent evolutionary tracks; and (iii) search for ongoing accretion activity and investigate the structure of the disk.

\section{VLT/X-shooter observations of B275}

VLT/X-shooter spectra were obtained of the massive YSO B275 in M $17\left(\right.$ CEN 24, RA $(2000.0)=18^{\mathrm{h}} 20^{\mathrm{m}} 25 \mathrm{~s} .13, \operatorname{Dec}(2000.0)=$ $-16^{\circ} 10^{\prime} 24^{\prime \prime} 56, V=15.55 \mathrm{mag}, K=8.05 \mathrm{mag}$, Chini et al. 1980; Skrutskie et al. 2006) on August 11, 2009 at 03h20 UT, during the first science verification run (PI Chini). The observations in the UVB arm $(300-600 \mathrm{~nm})$ were binned ( 2 pixels) in the wavelength direction in order to increase the signal-to-noise ratio of this part of the spectrum, while still oversampling the resolution element. The $1.6^{\prime \prime}$ slit was used resulting in resolving power $R=3300$. For the VIS $(550-1000 \mathrm{~nm})$ and the NIR arm $(1000-2500 \mathrm{~nm})$ a $0.9^{\prime \prime}$ slit was used $(R=8800$ and 5600 , respectively). The total exposure time was $45 \mathrm{~min}$, resulting in a typical signal-to-noise ratio of 70 . For more details on the $\mathrm{X}$-shooter instrument and its performance, see D'Odorico et al. (2006); Vernet et al. (2011). The observing conditions were good ( $0.6^{\prime \prime}$ seeing in $V$ and $76 \%$ Moon illumination). The spectra were obtained by nodding the star on the slit, allowing for background subtraction. The standard procedures of data reduction were applied using the X-shooter pipeline version 0.9.4 (Goldoni et al. 2006; Modigliani et al. 2010). For flux calibration and telluric absorption correction, the standard stars EG274 and HD 180699 were used.

\section{Results}

In the following we present the results for the accurate classification of the photospheric spectrum, analyze the interstellar spectrum to determine the extinction, model the SED using the fluxcalibrated X-shooter spectrum, and describe the emission-line spectrum produced by the circumstellar disk.

\subsection{Spectral classification}

Hydrogen absorption lines were detected by Hanson et al. (1997) in the blue spectrum of B275, but do not allow for an accurate spectral classification. As shown in Fig. 1, a number of helium and metal lines can be used to classify the photospheric spectrum. The He I $400.9 \mathrm{~nm}$ and C II $426.7 \mathrm{~nm}$, prominent down to spectral type B3, are very weak. The He I $447.1 \mathrm{~nm} / \mathrm{Mg}$ II $448.1 \mathrm{~nm}$ ratio is a useful spectral indicator for mid- to late-B stars (Gray \& Corbally 2009) as the neutral helium line disappears towards lower temperature (A0) and the magnesium line strengthens. When also considering another line ratio, Si II $412.8 \mathrm{~nm} / \mathrm{He}$ I $448.1 \mathrm{~nm}$, the spectral type becomes B6 ( \pm one subtype).

The spectral type and luminosity class of B275 are further constrained by comparison of the observed $\mathrm{H}$ I and $\mathrm{He} \mathrm{I}$ line profiles (as well as the shape of the SED, see Sect. 3.3), to model profiles produced with FASTWIND (Puls et al. 2005). This code calculates non-LTE line-blanketed stellar atmosphere models and is especially suited to modeling stars with strong winds, but it can also be used to examine $T_{\text {eff }}$ and $\log g$ dependent photospheric lines of $\mathrm{H}$ and $\mathrm{He}$. We constructed a grid of models (in varying $T_{\text {eff }}$ and $\log g$ ) of B6-B8 dwarf and giant stars. The synthetic $\mathrm{H}_{\mathrm{I}}$ and $\mathrm{He}$ I profiles resulting from the models are convolved with the corresponding instrumental and rotational profiles. We adopt $v_{\mathrm{r}} \sin i=100 \mathrm{~km} \mathrm{~s}^{-1}$. An acceptable fit is obtained for a B7 V model (Fig. 2); however, the best fit is obtained for a B7 III model, with $T_{\text {eff }}=13000 \pm 500 \mathrm{~K}$ and 


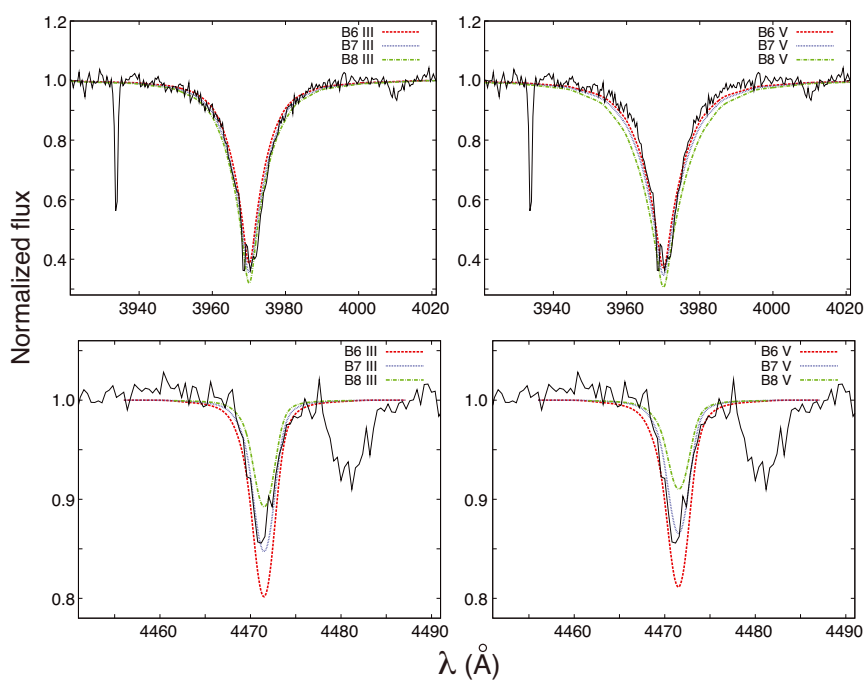

Fig. 2. FASTWIND model profiles of $\mathrm{H} \epsilon$ (top) and He I $447.1 \mathrm{~nm}$ (bottom) lines for B6-B8 giants (left) and main-sequence stars (right). The B7 III model provides the best fit with the observed profiles.

$\log g=3.5 \pm 0.3$. This is the first time that the spectral type of a candidate massive YSO has been accurately determined.

\subsection{Interstellar spectrum}

The optical spectrum of B275 includes several interstellar features: atomic resonance transitions (e.g., $\mathrm{Ca}$ II H\&K, Na I D) and diffuse interstellar bands (DIBs). The DIB strength provides a measure of the interstellar extinction. For the DIBs centered at $578.0,579.7$, and $661.4 \mathrm{~nm}$, we measure an equivalent width of $0.063,0.014$, and $0.021 \mathrm{~nm}$, respectively, with a typical error of $10 \%$. Using the relations from Cox et al. (2005), we arrive at an $E(B-V)$ of $1.0 \pm 0.1 \mathrm{mag}$. For an average value of $R_{V}=3.1$, these DIB strengths yield $A_{V} \simeq 3$ mag of visual extinction. This is less than the determination of $A_{V} \simeq 6.1 \mathrm{mag}$ from dereddening the SED (Sect. 3.3). Hanson et al. (1997) note that the DIB features in spectra of M 17 stars do not show large variations in strength, despite the fairly wide range in total extinction, from $A_{V}=3-10$ mag. We consider their explanation likely that the DIBs are mostly tracing the foreground dust and that the (unidentified) DIB carriers may only exist in the diffuse medium, not in the dark cloud environment of M 17.

\subsection{Spectral energy distribution}

Figure 3 shows the flux-calibrated X-shooter spectrum (300$2500 \mathrm{~nm}$ ) of B275. The photometric data points demonstrate the accuracy of the spectrophotometric calibration. The long standing debate over the distance to M 17 (ranging from 1.3 to $2.1 \mathrm{kpc}$ ) has recently been settled by the measurement of the trigonometric parallax of the $\mathrm{CH}_{3} \mathrm{OH}$ maser source G15.03-0.68 (Xu et al. 2011), resulting in a distance of $1.98_{-0.12}^{+0.14} \mathrm{kpc}$ so that M 17 is likely located in the Carina-Sagittarius spiral arm.

We deredden the flux-calibrated X-shooter spectrum of B275 (Fig. 3) using the parameterization of the extinction law by Cardelli et al. (1989). The dereddened spectrum is fit to a Kurucz model (Kurucz 1979, 1993) based on an iterative procedure, with fixed parameters $T_{\text {eff }}=13000 \mathrm{~K}, \log g=3.5, d=1.98 \mathrm{kpc}$ and $R_{V}=3.3$ (an effective value resulting from interstellar and local extinction). This yields independent best-fit values of $A_{V}=6.1 \pm$ $0.6 \mathrm{mag}$ and $R_{\star}=8.1 \pm 0.8 R_{\odot}$. Note that this radius is much

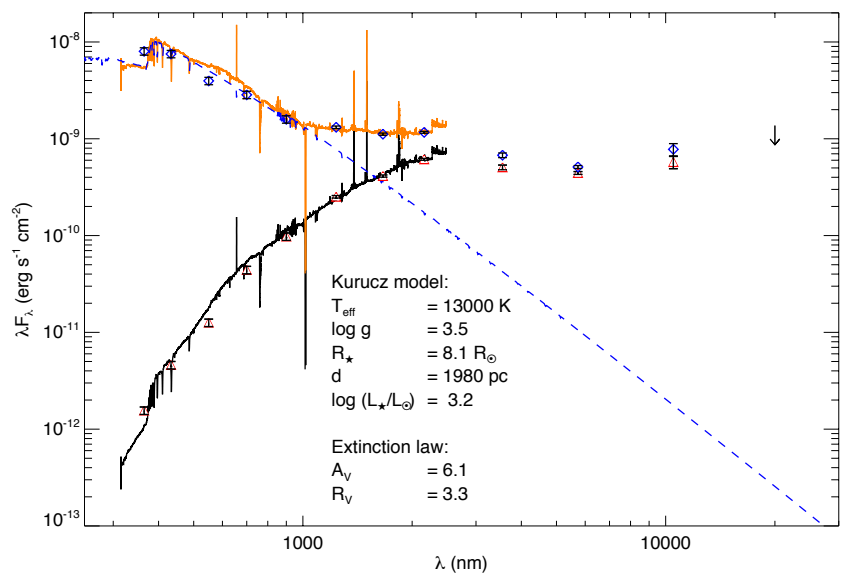

Fig. 3. The flux-calibrated X-shooter spectrum of B275 from 300-2500 nm (black) along with the photometric data (red triangles, black error bars) from Chini et al. (1980) (UVBRI), 2MASS (Skrutskie et al. 2006, JHK), Spitzer GLIMPSE (Benjamin et al. 2003, 3.6, and $5.8 \mu \mathrm{m})$, and Nielbock et al. (2001) (N, Q). When dereddened $\left(A_{V}=\right.$ $6.1 \mathrm{mag}$, orange line, blue diamonds), the SED is described well by a B7 III Kurucz model (blue, dashed line). The excess flux at 500-800 nm is an instrumental feature.

larger than that of, e.g., a B5 zero-age main sequence (ZAMS) star $\left(2.7 R_{\odot}\right.$, Hanson et al. 1997). An additional constraint is provided by the height of the Balmer jump, which also varies with $T_{\text {eff }}$ and $R_{\star}$ : Fig. 3 demonstrates that the Balmer jump (as well as the Paschen jump) is nicely fit to the observed spectrum. Thus, with a larger radius the discrepancy between the classification of the photospheric spectrum and the dereddened SED is solved. The consequence is that B275 is not on the main sequence but is a so-called bloated star, where the appropriate spectral type would be B7 III. The corresponding luminosity is $\log L_{\star} / L_{\odot}=3.2$.

\subsection{Accretion signatures}

A pronounced, double-peaked emission feature is detected in the strongest $\mathrm{H}$ Balmer lines, the Ca II triplet and the $\mathrm{O}_{\mathrm{I}} 844.6 \mathrm{~nm}$ line (Fig. 1). The measured peak-to-peak separation ranges from $71 \pm 7 \mathrm{~km} \mathrm{~s}^{-1}(\mathrm{O}$ II $844.6 \mathrm{~nm})$ to $105 \pm 3 \mathrm{~km} \mathrm{~s}^{-1}$ (Ca II $849.8 \mathrm{~nm}$ ), and is centered at the rest-frame velocity of the star. The $\mathrm{Ca}$ II triplet lines are probably produced in an optically thick medium, since their strength ratio is not 1:9:5. The strongest lines of the $\mathrm{H}$ I Paschen and Brackett series also exhibit a central emission component, though it is single-peaked. The higher series members include a weaker emission component that may be double-peaked. A number of metallic emission lines (e.g., C I and Fe II) are detected throughout the spectrum, albeit very weak.

Prominent CO 1st-overtone emission bandheads are detected at $2.3 \mu \mathrm{m}$, with clear evidence of a blue shoulder. We also confirm the presence of 2 nd-overtone $\mathrm{CO}$ bandhead emission at $1.5 \mu \mathrm{m}$ (Hanson et al. 1997). CO is easily dissociated so must be shielded from the strong UV flux of the young massive star. On the other hand, to produce 1st overtone emission, CO must be excited, requiring a temperature in the range between 1500 and $4500 \mathrm{~K}$ (Bik \& Thi 2004). This temperature might even be higher, considering the unprecedented detection of 2 nd overtone emission. These conditions can be met in the plane of a dense circumstellar disk where the $\mathrm{CO}$ molecules can be formed, excited, and protected from dissociation through self-shielding. 


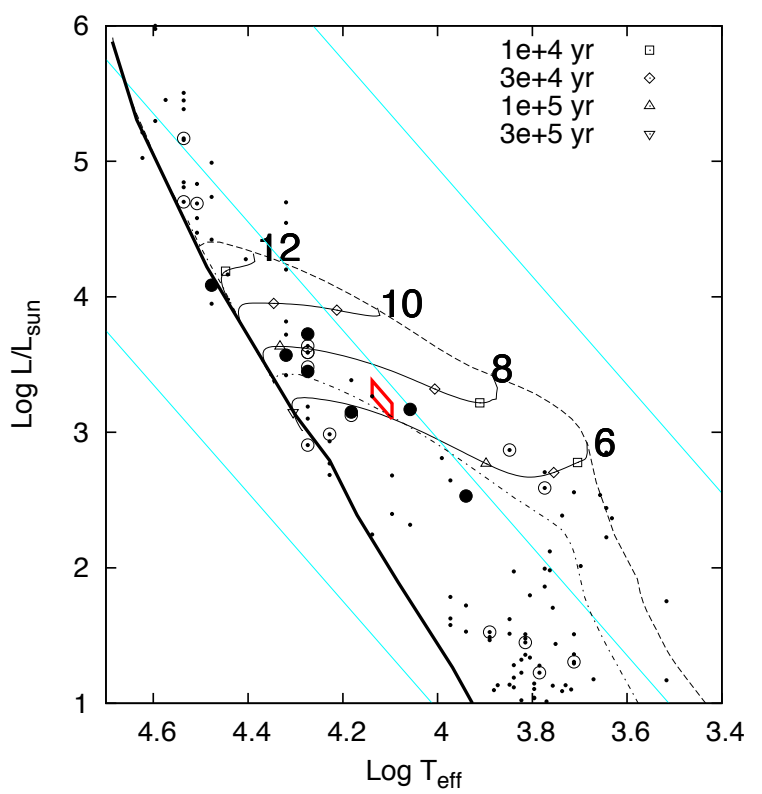

Fig. 4. The location of B275 (red parallellogram) in the HRD next to PMS tracks from Hosokawa \& Omukai (2009) with the ZAMS mass labeled and open symbols indicating lifetimes. The thin dashed and thin dot-dashed lines are the birth lines for accretion rates of $10^{-4} M_{\odot} \mathrm{yr}^{-1}$ and $10^{-5} M_{\odot} \mathrm{yr}^{-1}$, respectively; the thick solid line is the ZAMS (Schaller et al. 1992). The filled and open circles represent stars in M 17 for which a spectral type has been determined (Hoffmeister et al. 2008), within a radius of 0.5 and 1.0, respectively; dots are other stars in M 17. B275 is on its way to becoming a 6-8 $M_{\odot}$ ZAMS star.

The relative strength and shape of the $\mathrm{CO}$ bandheads can be modeled by an optically thin Keplerian disk (Bik \& Thi 2004; Blum et al. 2004), where the blue shoulder would imply a relatively high inclination angle of the disk ("edge-on"). Blum et al. (2004) model the CO 2-0 first-overtone ro-vibrational bandhead at $2294 \mathrm{~nm}$ of B275 resulting in $v \sin i=109.7 \pm 0.6 \mathrm{~km} \mathrm{~s}^{-1}$ (at the inner edge of the $\mathrm{CO}$ emission zone) and surface density $N_{\mathrm{CO}}=3.5 \pm 0.2 \times 10^{21} \mathrm{~cm}^{-2}$. The double-peaked emission profiles, as shown in Fig. 1, are very similar to the emission-line profile of a single line obtained by Blum et al. (2004).

We find no evidence for veiling of the optical spectrum or any strong indications of active "heavy" accretion and/or jets, such as those observed in some other systems (e.g., Ellerbroek et al. 2011). The [O II] $630 \mathrm{~nm}$ line very likely has a nebular origin.

\section{Discussion}

The accurate spectral classification and SED fit result in a welldefined position of B275 in the Hertzsprung-Russell diagram (HRD, Fig. 4). It is located well above the ZAMS, demonstrating its PMS nature. If B275 is contracting towards the ZAMS, the final ZAMS mass would be $6-8 M_{\odot}$ (spectral type B1-B2), assuming that no additional mass is accreted.

To be visible at this location in the HRD, the star must have experienced an average accretion rate of at least $10^{-5} M_{\odot} \mathrm{yr}^{-1}$ in its recent history. B275 may thus be the long-sought-for example of an early-B PMS star. Figure 4 also shows other nearby stars in M 17. Hanson et al. (1997) derive an age of $\sim 1$ Myr for the M 17 cluster andHoffmeister et al. (2008) estimate that the
PMS objects are less than $5 \times 10^{5} \mathrm{yr}$ old. Based on its location on the HRD we estimate the age of B275 at $10^{5} \mathrm{yr}$.

B275 bears some resemblance to a classical Be star. However, we note that Be stars do not emit CO 1st and 2nd overtone emission. In addition, B275 would be classified as a luminous Herbig Be star according to the definition discussed in Carmona et al. (2010), but our analysis of the photospheric spectrum allows for a more quantitative classification.

B275 has a significant amount of infrared excess, starting at $1 \mu \mathrm{m}$, and a flat SED between 2 and $10 \mu \mathrm{m}$ (Nielbock et al. 2001). This indicates the presence of a flaring circumstellar disk in which the dust has not settled yet. However, the visibility of the photosphere, the small number of optical gas emission lines and the absence of a jet lead us to believe that the current mass-accretion rate is not very high. Nevertheless, the $\mathrm{CO}(2-0)$ and (3-0) emission originates in a dense and highly excited inner part of the disk. Either the system is in an intermittent phase between accretion episodes, or it is on the verge of photo-evaporating its disk. Either scenario is consistent with its location in the HR-diagram: an intermediate-mass, visible star in M 17 on its way to becoming an early-B main-sequence star.

Acknowledgements. We thank Takashi Hosokawa for kindly providing the PMS tracks. The ESO Paranal staff is acknowledged for obtaining the X-shooter spectrum of B275. We thank the anonymous referee for useful comments and suggestions.

\section{References}

Appenzeller, I., \& Mundt, R. 1989, A\&ARv, 1, 291

Benjamin, R. A., Churchwell, E., Babler, B. L., et al. 2003, PASP, 115, 953 Bik, A., \& Thi, W. F. 2004, A\&A, 427, L13

Bik, A., Kaper, L., \& Waters, L. B. F. M. 2006, A\&A, 455, 561

Blum, R. D., Barbosa, C. L., Damineli, A., Conti, P. S., \& Ridgway, S. 2004, ApJ, 617, 1167

Broos, P. S., Feigelson, E. D., Townsley, L. K., et al. 2007, ApJS, 169, 353

Cardelli, J. A., Clayton, G. C., \& Mathis, J. S. 1989, ApJ, 345, 245

Carmona, A., van den Ancker, M. E., Audard, M., et al. 2010, A\&A, 517, A67

Chini, R., Elsaesser, H., \& Neckel, T. 1980, A\&A, 91, 186

Chini, R., Hoffmeister, V., Kimeswenger, S., et al. 2004, Nature, 429, 155

Cox, N. L. J., Kaper, L., Foing, B. H., \& Ehrenfreund, P. 2005, A\&A, 438, 187

D'Odorico, S., Dekker, H., Mazzoleni, R., et al. 2006, in SPIE Conf. Ser., 6269

Ellerbroek, L. E., Kaper, L., Bik, A., et al. 2011, ApJ, 732, L9

Goldoni, P., Royer, F., François, P., et al. 2006, in SPIE Conf. Ser., 6269

Gray, R. O., \& Corbally, J., C. 2009, Stellar Spectral Classification (Princeton University Press)

Hanson, M. M., Howarth, I. D., \& Conti, P. S. 1997, ApJ, 489, 698

Hanson, M. M., Luhman, K. L., \& Rieke, G. H. 2002, ApJS, 138, 35

Hoffmeister, V. H., Chini, R., Scheyda, C. M., et al. 2008, ApJ, 686, 310

Hosokawa, T., \& Omukai, K. 2009, ApJ, 691, 823

Hosokawa, T., Yorke, H. W., \& Omukai, K. 2010, ApJ, 721, 478

Keto, E., Broderick, A. E., Lada, C. J., \& Narayan, R. 2006, ApJ, 652, 1366

Kraus, S., Hofmann, K., Menten, K. M., et al. 2010, Nature, 466, 339

Krumholz, M. R., Klein, R. I., McKee, C. F., Offner, S. S. R., \& Cunningham, A. J. 2009, Science, 323, 754

Kurucz, R. L. 1979, ApJS, 40, 1

Kurucz, R. L. 1993, VizieR Online Data Catalog, 6039, 0

Modigliani, A., Goldoni, P., Royer, F., et al. 2010, in SPIE Conf. Ser., 7737

Nielbock, M., Chini, R., Jütte, M., \& Manthey, E. 2001, A\&A, 377, 273

Povich, M. S., Churchwell, E., Bieging, J. H., et al. 2009, ApJ, 696, 1278

Puls, J., Urbaneja, M. A., Venero, R., et al. 2005, A\&A, 435, 669

Schaller, G., Schaerer, D., Meynet, G., \& Maeder, A. 1992, A\&AS, 96, 269

Skrutskie, M. F., Cutri, R. M., Stiening, R., et al. 2006, AJ, 131, 1163

Testi, L., Tan, J. C., \& Palla, F. 2010, A\&A, 522, A44

Urquhart, J. S., Moore, T. J. T., Hoare, M. G., et al. 2011, MNRAS, 410, 1237

Vernet, J., Dekker, H., D’Odorico, S., et al. 2011, A\&A, in press,

DOI: $10.1051 / 0004-6361 / 201117752$

Wheelwright, H. E., Oudmaijer, R. D., de Wit, W. J., et al. 2010, MNRAS, 408, 1840

Xu, Y., Moscadelli, L., Reid, M. J., et al. 2011, ApJ, 733, 25 